\title{
Estimation of Sequence Distribution in Poly(vinyl acetate)
}

\author{
Tohei Yamamoto, Teizo Isono, ${ }^{*}$ Teruya Matsumoto, \\ Mitsuhiro Sugimoto, Osamu Sangen, \\ and Mikiharu KAMACHI ${ }^{* *}$ \\ Faculty of Engineering, Himeji Institute of Technology, \\ Shosha, Himeji 671-22, Japan \\ * Hyogo Prefectural Institute of Industrial Technology, \\ Nomura, Nishiwaki 677, Japan \\ ** Faculty of Science, Osaka University, Machikaneyama, \\ Toyonaka 560, Japan
}

(Received March 25, 1994)

\begin{abstract}
KEY WORDS Sequence Distribution / Computer Simulation / Random Numbers / Monte Carlo Method / Poly(vinyl acetate) /
\end{abstract}

We found a good method to saponify poly(vinyl ester)s with bulky ester groups and determined their tacticities by ${ }^{1} \mathrm{H}$ NMR spectrum of resulted poly(vinyl alcohol) (PVA). ${ }^{1}$ The tacticities of poly(vinyl ester)s were found to vary with bulkiness of ester groups $^{2}$ and cotacticities of copolymers of vinyl acetate (VAc) with vinyl propionate (VPr) ${ }^{3}$ vinyl decanoate $(\mathrm{VD})^{4}$ and vinyl pivalate $(\mathrm{VP})^{5}$ varied with feed monomer composition. Although cotacticities were not well simulated by the terminal model for copolymers of VAc with VP indicating some penultimate effects, they were well simulated for copolymers of VAc with VPr and VD by the terminal model. ${ }^{6}$ Hence, we consider that homo-polymerizations of VAc, VPr, and VD can be explained by the terminal model and sequences in these polymers can be estimated by random numbers (Sequence distribution in copolymer of styrene with $\alpha$-methyl styrene, etc. were simulated by Monte Carlo $\operatorname{method}^{7}$ ). The present paper reports estimation of the sequence distribution in poly(vinyl acetate) (PVAc) using random numbers with drand 48 in the UNIX system. The sequences can be estimated by Bernoulli's statistics, but we consider that the Monte Carlo method has some merits. For example, sequence distributions estimated can be drawn as shown in Figure 1 and the esimation can be carried out for $P_{n}$ as shown in Table I.

In the case of PVAc with $P_{n}=1000$, random numbers of 1-1000 were generated by a computer. The polymerization of VAc obeys the terminal model and the isotactic parameter $(\sigma)$ is 0.474 . Thus random numbers of $1-474$ were assigned to meso diad, and those of $475-1000$ to racemo diad. Seqence distributions were determined and an example is shown in Figure 1.

As shown in the figure, long sequences as $r_{11}$ and $m_{8}$ could be observed. The frequencies of $r$ and $m$ were counted, and the ratios of $r / m$, $\mathrm{rr} / \mathrm{mm}$ and $\mathrm{rrr} / \mathrm{mmmm}$ were calculated. The procedures were repeated $10^{7} / P_{n}$ times and the ratios were averaged. The values for 5 examples and the average values are listed in Table I.

Sequence distributions for other $P_{n}$ were also determined in the same manner, and the ratios were calculated as shown in Table I. The ratios determined by Bernoulli's statistics are listed in Table I. 
r m r r r m m m r m m r r m r r m m r m r r m r m r r m m m mm r $-\mathrm{mmr} \mathrm{rmmr} r \mathrm{rmr} r \mathrm{rmmmr} \mathrm{rmr} r \mathrm{r} \mathrm{rmmmmrmmmrmm}$ $\rightarrow \mathrm{mmmrmmmrmrmmrmmmrrrrmm} \mathrm{rmm}$ -mmmrrmrrmmmmrmrmmmmmmrrmmr rm rmm rm $\rightarrow \mathrm{rmrrmrrrrrrmmmrrmm} \mathrm{r} \mathrm{r} \mathrm{m} \mathrm{r} \mathrm{m} \mathrm{r} \mathrm{m} \mathrm{r} \mathrm{r} \mathrm{m} \mathrm{m} \mathrm{m} \mathrm{m} \mathrm{m}$ - rmrrrmrrmmrrmrrrmmrrrrm rmm

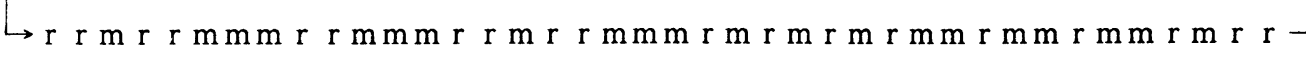
- mmmmrmrrrrrrmm r r r m r m r m r r m r m r m $\rightarrow \mathrm{rrrrrrrrrmmrmmmrmm} \mathrm{rmm} \mathrm{rmm}_{-}$ -rmrmrmrmrrrmmmrmrmmrmmrrrmrmrrmm rm

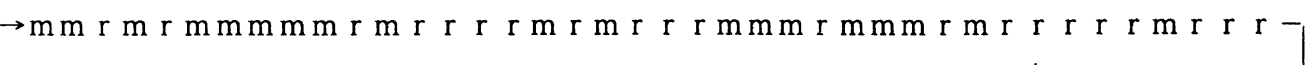

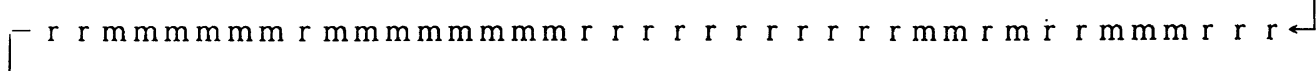
$\rightarrow$ rmmmmmrmrrmmm r rmrmrrmmm rmmmmmrmm m m m - r r r r r r m r m rmmr rmmr r r r m r r r m rm r r r r m $\rightarrow \mathrm{mrmrmr} r \mathrm{~mm} \mathrm{rm}$ rmr rmmmr m r m r m r r rmm mmm r[ r m m r m r m m r r r r m r m mmmrmmrmrr rmm mmm $\rightarrow \mathrm{mrr}$ r m r m r mmmmmrrrmm r m r m r m r m r m r - r r r r m rmmmrmmrmr rmmmr m rmmrmrrrmrrrr

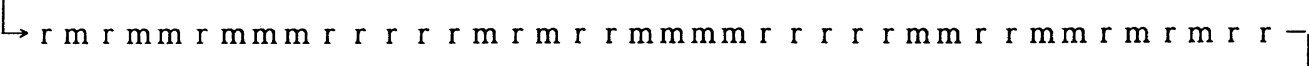

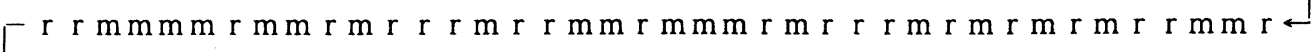

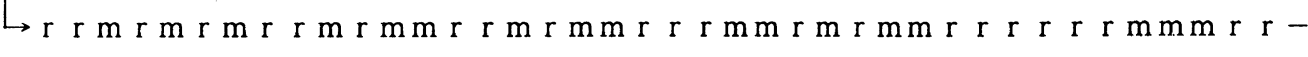
- mmrmrmrr rmmmrmrrrmrmrmrmrrmrmmr rm rmm $\hookrightarrow \mathrm{mmmmrmrmrmmmmrrmmrmmrmrmm}$ - m r m r r m r m r mmmmrmrmmrmmmmrmrmmm r r r r r m m

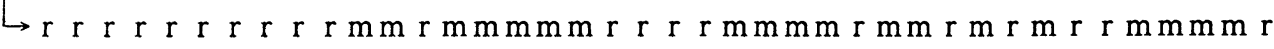

Figure 1. Example of sequence distribution in poly $(\mathrm{VAc})$ obtained by radical polymerization at $0^{\circ} \mathrm{C}$. $P_{n}=1000 ; \sigma=0.474 ; \mathrm{r} / \mathrm{m}=1.06 ; \mathrm{rr} / \mathrm{mm}=1.28 ; \mathrm{rrr} / \mathrm{mmmm}=1.59$.

Good reproducibility in the ratios and good agreement of ratios obtained by Monte Carlo and Bernoulli's were found for high $P_{n}$ $(>1000)$, but reproducibility of the ratios, especially $\mathrm{rrr} / \mathrm{mmmm}$, was not good for low $P_{n}(\leqq 1000)$. These results suggest that sequence distributions in each PVAc molecule with small $P_{n}$ are quite different. However, the average values of $\mathrm{rrr} / \mathrm{mmmm}$ for PVAc with respective $P_{n}$, except $P_{n}=100$, are not so much different from each other. Values of $r / m$ and $r r / m m$ were in good agreement with those determined by ${ }^{1} \mathrm{H}$ NMR spectrum. Values of $\mathrm{rrr} / \mathrm{mmmm}$ were a little different from those determined by the ${ }^{13} \mathrm{C}$ NMR spectrum (we consider that the difference may be caused by penultimate effect). The sequence distributions generated by computer are considered to represent approximately those in actual PVAc.

We consider that such long racemo sequences as $r_{11}$ are important factors for the properties of PVA such as gelation of PVA aq solution. We are investigating the sequence distributions of the other poly(vinyl ester)s and the results will be reported in the near future. 
Table I. Estimated ratios of racemo and meso sequences in PVAc obtained by radical polymerization at $0^{\circ} \mathrm{C}$

\begin{tabular}{|c|c|c|c|c|}
\hline Run & $P_{n}$ & $r / m$ & $\mathrm{rr} / \mathrm{mm}$ & $\mathrm{rrrr} / \mathrm{mmmm}$ \\
\hline $\mathrm{Al}$ & 100 & 0.852 & 0.727 & 0.385 \\
\hline $\mathrm{A} 2$ & & 0.887 & 0.762 & 0.400 \\
\hline A3 & & 1.50 & 2.12 & 15.0 \\
\hline A4 & & 0.961 & 0.923 & 0.750 \\
\hline A5 & & 1.33 & 2.00 & $\infty$ \\
\hline- & & - & - & - \\
\hline Average $^{a}$ & & $1.13(0.232)$ & $1.36(0.608)$ & \\
\hline $\mathrm{B} 1$ & 500 & 1.06 & 1.11 & 1.06 \\
\hline B2 & & 0.916 & 0.843 & 0.882 \\
\hline B3 & & 1.08 & 1.06 & 1.35 \\
\hline B4 & & 1.03 & 1.06 & 0.939 \\
\hline B5 & & 1.11 & 1.25 & 1.70 \\
\hline - & & - & - & - \\
\hline Average $^{a}$ & & $1.11(0.101)$ & $1.25(0.230)$ & $1.70(0.877)$ \\
\hline $\mathrm{Cl}$ & 1000 & 1.07 & 1.13 & 1.59 \\
\hline $\mathrm{C} 2$ & & 1.21 & 1.46 & 2.74 \\
\hline $\mathrm{C} 3$ & & 1.03 & 1.07 & 1.43 \\
\hline $\mathrm{C} 4$ & & 1.04 & 1.08 & 1.04 \\
\hline $\mathrm{C} 5$ & & 1.03 & 1.06 & 1.17 \\
\hline - & & - & - & - \\
\hline Average $^{a}$ & & $1.11(0.070)$ & $1.24(0.158)$ & $1.60(0.512)$ \\
\hline D1 & 5000 & 1.09 & 1.18 & 1.63 \\
\hline D2 & & 1.15 & 1.34 & 1.74 \\
\hline D3 & & 1.07 & 1.14 & 1.15 \\
\hline D4 & & 1.08 & 1.16 & 1.29 \\
\hline D5 & & 1.10 & 1.21 & 1.50 \\
\hline- & & - & -- & - \\
\hline Average $^{a}$ & & $1.11(0.032)$ & $1.23(0.071)$ & $1.53(0.211)$ \\
\hline El & 10000 & 1.12 & 1.25 & 1.53 \\
\hline $\mathrm{E} 2$ & & 1.09 & 1.20 & 1.35 \\
\hline E3 & & 1.10 & 1.22 & 1.29 \\
\hline E4 & & 1.10 & 1.22 & 1.54 \\
\hline E5 & & 1.15 & 1.33 & 1.83 \\
\hline- & & - & - & - \\
\hline
\end{tabular}

Table I. (continued.)

\begin{tabular}{|c|c|c|c|c|}
\hline Run & $P_{n}$ & $r / m$ & $\mathrm{rr} / \mathrm{mm}$ & $\mathrm{rrrr} / \mathrm{mmmm}$ \\
\hline Average & & $1.11(0.023)$ & $1.23(0.051)$ & $1.52(0.149)$ \\
\hline Found & 7870 & $1.11^{\mathrm{b}}$ & $1.23^{\mathrm{b}}$ & $1.75(0.0082)^{\mathrm{c}}$ \\
\hline Calculate & & 1.11 & 1.23 & 1.52 \\
\hline \multicolumn{5}{|c|}{$\begin{array}{l}\text { From } 10^{7} / P_{n} \text { times (one cycle of drand } 48 \text { ) calculation. } \\
\text { Values in ( ) are standard deviations. } \\
\text { b Measured by }{ }^{1} \mathrm{H} \text { NMR spectrum of PVA derived from } \\
\text { PVAc. } \\
\text { c Measured by }{ }^{13} \mathrm{C} \text { NMR spectrum of PVA derived } \\
\text { from PVAc. Value in ( ) is standard deviation. } \\
\text { d Calculated by Bernoulli's statistics. }\end{array}$} \\
\hline
\end{tabular}

\section{REFERENCES}

1. T. Yamamoto, S. Yoda, H. Takase, T. Saso, O. Sangen, R. Fukae, T. Sato, and M. Kamachi, Polym. J., 23, 185 (1991).

2. T. Yamamoto, Y. Hasegawa, J. Teragaki, O. Sangen, R. Fukae, and M. Kamachi, Chem. Express, 4, 829 (1989).

3. T. Yamamoto, M. Hanatani, K. Hirota, H. Kishibe, O. Sangen, R. Fukae, M. Kamachi, and T. Sato, Kobunshi Ronbunshu, 50, 223 (1993).

4. T. Yamamoto, Y. Hasegawa, J. Teragaki, O. Sangen, R. Fukae, M. Kamachi, T. Sato, and Y. Fukunishi, Chem. Express, 6, 865 (1991)

5. T. Yamamoto, R. Fukae, T. Saso, O. Sangen, M. Kamachi, T. Satoh, and Y. Fukunishi, Polym. J., 24, 115 (1992).

6. T. Yamamoto, M. Hanatani, T. Matsumoto, O. Sangen, T. Isono, R. Fukae, and M. Kamachi, Polym. J., 26, 417 (1994).

7. I. Motoc and I. Muscutariu, J. Polym. Sci., Chem. Ed., 18, 757 (1980); A. Dwork, H. J. Harwood, and W. J. Freeman, Polym. J., 17, 351 (1985); K. Kamide, and K. Hisatani, Polym. Prepr. Jpn., 39, 3851 (1990). 